\title{
Modeling Stem Cell Asymmetry in Yeast
}

\author{
P.H. ThORPE, ${ }^{*}$ J. BRUNo,$^{\dagger}$ AND R. ROTHSTEIN* \\ *Department of Genetics and Development, Columbia University Medical Center, New York, \\ New York 10032; ${ }^{\dagger}$ Massachusetts Institute of Technology, Cambridge, Massachusetts 02139
}

\begin{abstract}
For adult stem cells to both self-renew and give rise to differentiating progenitors, they must undergo an inherently asymmetric division. This defining model of asymmetric cell division requires either that stem cells preferentially distribute internal factors, thereby maintaining a stem cell phenotype in one lineage, or that extrinsic signals determine the fate of daughter cells, allowing the maintenance of one stem cell lineage. Although microbial systems are often used to model asymmetry, lineagespecific asymmetry has not been characterized in these organisms. Recently, we identified a stem-cell-like lineage-specific pattern of kinetochore asymmetry in postmeiotic yeast spores. Because the function of the kinetochore is to segregate chromosomes, this asymmetry has the potential to segregate sister chromatids nonrandomly. This may be relevant to stem cells because more than 30 years ago, it was proposed that stem cells selectively segregate one strand of their chromosomes into the selfrenewing stem cell lineage (Cairns 1975). Although advanced labeling methods have provided evidence to both support and refute this hypothesis, it remains unclear how nonrandom sister-chromatid segregation might be achieved in a stem cell lineage. We have identified a kinetochore-specific mechanism in yeast that could support lineage-specific nonrandom sister-chromatid segregation and we discuss the implications of this observation.
\end{abstract}

Adult stem cells exhibit a lineage-specific asymmetric division pattern. When each stem cell divides, it generates a new stem cell and a progenitor cell, the latter ultimately giving rise to new cells in the tissue. This standard model of adult stem cell division does not preclude symmetric division, for example, to expand a stem cell pool. However, the asymmetric division provides a means for a stable population of stem cells to be maintained while enabling the creation of progenitor cells to replace damaged or aging cells within a tissue. One interesting consequence of this model, illustrated in Figure 1 (top), is that the stem cell lineage, represented by shaded cells, is a unique single lineage within a dividing population.

There are a number of microbial models of cellular asymmetry, but in these, the asymmetries are not confined to a single lineage and are found throughout the population. In Saccharomyces cerevisiae, every cell division is asymmetric, producing a mother and bud that have distinct fates. This inequality allows factors (e.g., alleles or protein complexes) to go into either the mother or the bud. An example of this asymmetry is illustrated by the RNA encoding Ash1, an inhibitor of the $H O$ endonuclease, which is specifically transported to the bud rather than the mother (Long et al. 1997). This results in buds being unable to switch mating type, unlike mother cells that can switch. This pattern of asymmetry is illustrated in Figure 1 (bottom), with cells capable of mating-type switching indicated by shading.

Using a strategy that involves fluorescently tagged proteins, we identified a lineage-specific asymmetry in the segregation of yeast kinetochore proteins. The asymmetric pattern is strikingly similar to the asymmetric model of adult stem cell division (Fig. 1, top). This phenotype only occurs immediately after meiosis in the mother lineage
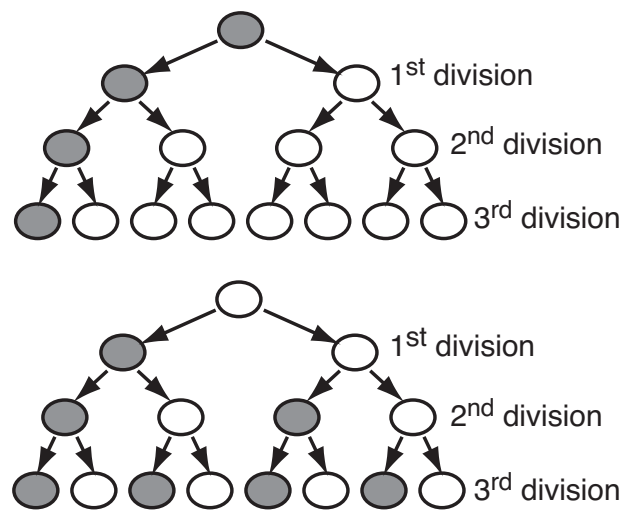

Figure 1. Two kinds of asymmetric divisions. (Top) Lineage-specific pattern of asymmetry seen in adult stem cells. Each division of the stem cell (shaded) gives rise to both a stem cell and progenitor cell (white). In contrast, progenitors give rise only to progenitors. This results in a single lineage of the stem cell in a given pedigree. This lineage contrasts with other patterns of asymmetry such as mating-type switching of $S$. cerevisiae shown below (bottom). In mating-type switching, a cell gives rise to both a switchable mother cell (shaded) and a nonswitching bud (white); however, this pattern is repeated at each division and therefore does not establish a single lineage.

that is derived from each spore (P. Thorpe et al., unpubl.). We have extended these studies and report the asymmetric segregation of a protein component of the spindle pole body, the yeast microtubule-organizing center. The kinetochore and spindle proteins direct segregation of sister chromatids during meiosis, and we discuss the implications of this phenotype with respect to potential nonrandom segregation of sister chromatids. 


\section{A NEW METHOD TO TRACK NONENCODED PROTEIN}

Because it is an excellent model for many aspects of basic cellular functions, we set out to discover a lineagespecific asymmetric program in yeast. We reasoned that such a program may be established when a new yeast strain is generated. This happens only twice in the normal yeast life cycle: when two haploid cells fuse to form a diploid cell and when a diploid cell undergoes meiosis to generate four new haploid spores. We examined meiosis because the transition from a diploid to haploid cell allowed us to use a straightforward strategy to follow protein segregation. We created haploid strains that have a given gene fused to a sequence encoding either yellow fluorescent protein (YFP) or cyan fluorescent protein (CFP). Two haploids were mated to form a diploid strain that was "heteroallelically" tagged for individual genes (YFP/CFP). This diploid strain was induced to undergo meiosis, generating haploid spores that inherited either the YFP- or CFP-tagged allele. Importantly, however, these spores also inherited both of the tagged proteins from the diploid parent, one of which is no longer genetically encoded (the method is illustrated in Fig. 2). Using time-lapse fluorescence microscopy, we specifically assayed the fluorescent protein that was no longer genetically encoded. By assaying the levels of nonencoded fluorescent protein, we avoid the confounding effects of transcription in these studies.

\section{SEGREGATION OF THE HISTONE HTA1}

We first examined histone H2A, encoded by HTA1. For this analysis, we created a diploid strain that is heteroallelically tagged, HTA1-YFP/HTA1-CFP, and induced this strain to undergo meiosis. Figure 3 shows an example of the resulting time-lapse sequences; levels of fluorescence within the nucleus at each division were assessed for each cell and the dilution of the nonencoded (YFP-tagged) pro- tein is evident. We found that the amount of nonencoded (or indeed total) fluorescence in the nucleus of the cells was equivalent for the mother and bud at each cell division. For nonencoded protein, we calculated the ratio of the fluorescence in the mother versus the bud - the mother-to-bud ratio $(\mathrm{m} / \mathrm{b})$ - to indicate if there was any deviation from parity. An $\mathrm{m} / \mathrm{b}$ ratio of 1 indicates equal fluorescence in the mother and bud after cell division. We found that the $\mathrm{m} / \mathrm{b}$ ratio of nonencoded Hta1 is 0.9 (standard error of the mean [S.E.M.] $=0.05$, standard deviation [S.D.] $=0.27$ ), indicating that approximately equal amounts of protein segregate to the mother and bud during cell division. Using the timelapse sequences, we monitored all of the divisions from a single spore, allowing us to create a pedigree and examine each separate cell division in the lineage. Figure 4 shows the first three divisions of spores and includes, with each, the mean $\mathrm{m} / \mathrm{b}$ ratio for Hta1. It is clear that the $\mathrm{m} / \mathrm{b}$ ratio for Htal is the same in each of the cell divisions examined.

We found that other proteins also show the same pattern of segregation in the spore and its progeny. These include Rad52, a DNA-repair and recombination protein, and an artificially engineered construct that consists of the tetracycline repressor fused to red fluorescent protein (TetRRFP), which binds to an array of Tet operator sequences located on yeast chromosome III (data not shown). The TetR-RFP fusion protein forms a focus specifically at a tandem array of operator sequences, and in this case, we specifically measured the fluorescence of each focus, rather than that of the whole nucleus.

\section{ASYMMETRIC SEGREGATION OF KINETOCHORE PROTEINS}

Interestingly, we found a different pattern of protein segregation when we examined four different kinetochore proteins. Ask1, Ctf19, Ndc10, and Mtw1 are each members of separate kinetochore subcomplexes (Cheeseman et al.

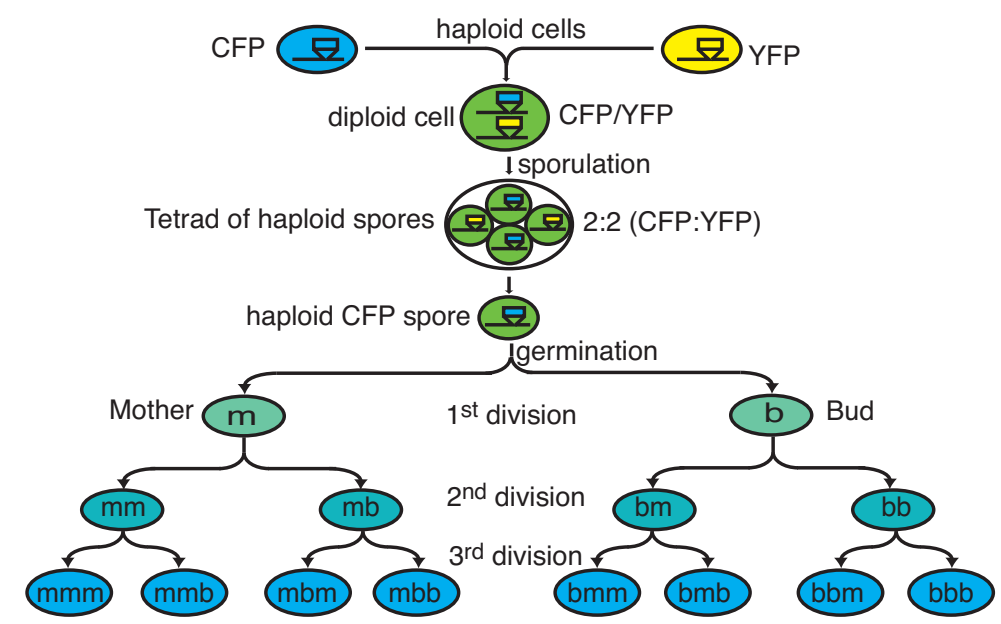

Figure 2. A labeling strategy for assaying nonencoded protein segregation. Two haploid strains containing the same gene tagged with different fluorescent proteins (YFP and CFP) are mated together to create a heteroallelically tagged strain. Because this diploid expresses both yellow- and blue-tagged protein, the cell is illustrated as green. This diploid is induced to undergo meiosis, producing four haploid spores that contain only one of the tagged alleles, but both proteins. An example of a spore inheriting the CFP-tagged allele is shown. As this cell undergoes successive divisions, the nonencoded yellow-tagged protein is diluted away and replaced with exclusively blue-tagged protein, illustrated as a transition from green to blue. 


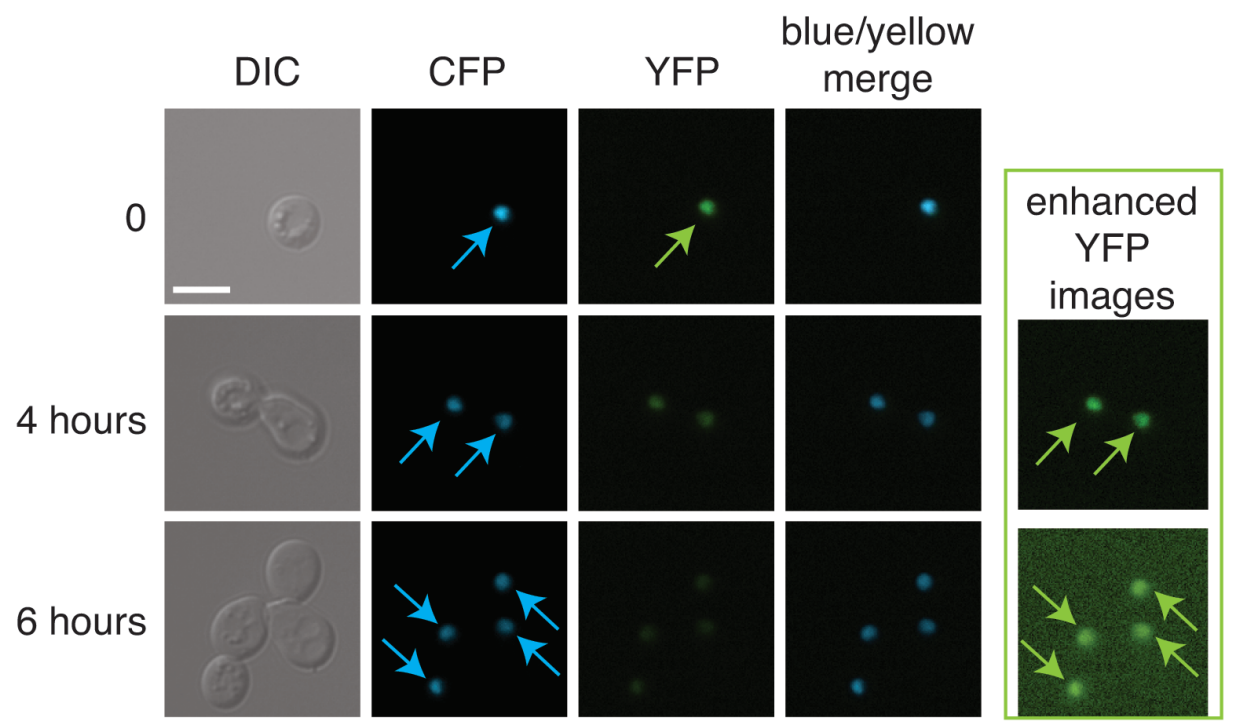

Figure 3. Microscopy images of a yeast spore encoding the tagged histone Hta1-CFP. Successive time-lapse images are arranged from top to bottom. The images in the first column are differential interference contrast (DIC) showing the spore and its progeny (bar, $5 \mu \mathrm{m}$ ). The next two columns, CFP and YFP, show the blue and yellow fluorescent channels, respectively, with the tagged histones in the nuclei of the cells indicated with arrows. These fluorescent images were deconvolved from a stack of vertically separated fluorescent exposures and are equivalently and uniformly enhanced. The fourth column shows a merge of the two fluorescent images. In the later time points $(4$ and 6 hours), the yellow-tagged histone becomes diluted, because it is no longer genetically encoded. However, to show that the protein is still detectable, in the fifth column, the YFP images have been artificially enhanced and the YFP-tagged histone indicated with arrows.

2001; De Wulf et al. 2003; Nekrasov et al. 2003; Pinsky et al. 2003; Bouck and Bloom 2005) and their concentrations can be quantified using fluorescent imaging (Joglekar et al. 2006). These proteins all localize to a single kinetochore focus in yeast, and it is the fluorescence of these foci that we used to calculate $\mathrm{m} / \mathrm{b}$ ratios (as for the TetR-RFP analysis described above). All four of these proteins show an asymmetric pattern of segregation that is confined to the spore and the mother lineage derived from it (Fig. 4) (P.
Thorpe et al., unpubl.). On average, we see twice as much protein in the mother cell versus the bud in this asymmetric mother lineage. Bud cells do not show this asymmetry nor do mother cells that are not directly descended from the spore. For example, nonencoded $\mathrm{Ndc10}$ has a mean $\mathrm{m} / \mathrm{b}$ ratio of 2.5 in the mother lineage (S.E.M. $=0.31$, S.D. $=1.8$ ) compared with a mean $\mathrm{m} / \mathrm{b}$ ratio of 1.2 in the other lineages (S.E.M. $=0.1$, S.D. $=0.7$ ). To assess whether this phenotype was specific to postmeiotic cells, we used time-lapse fluo-

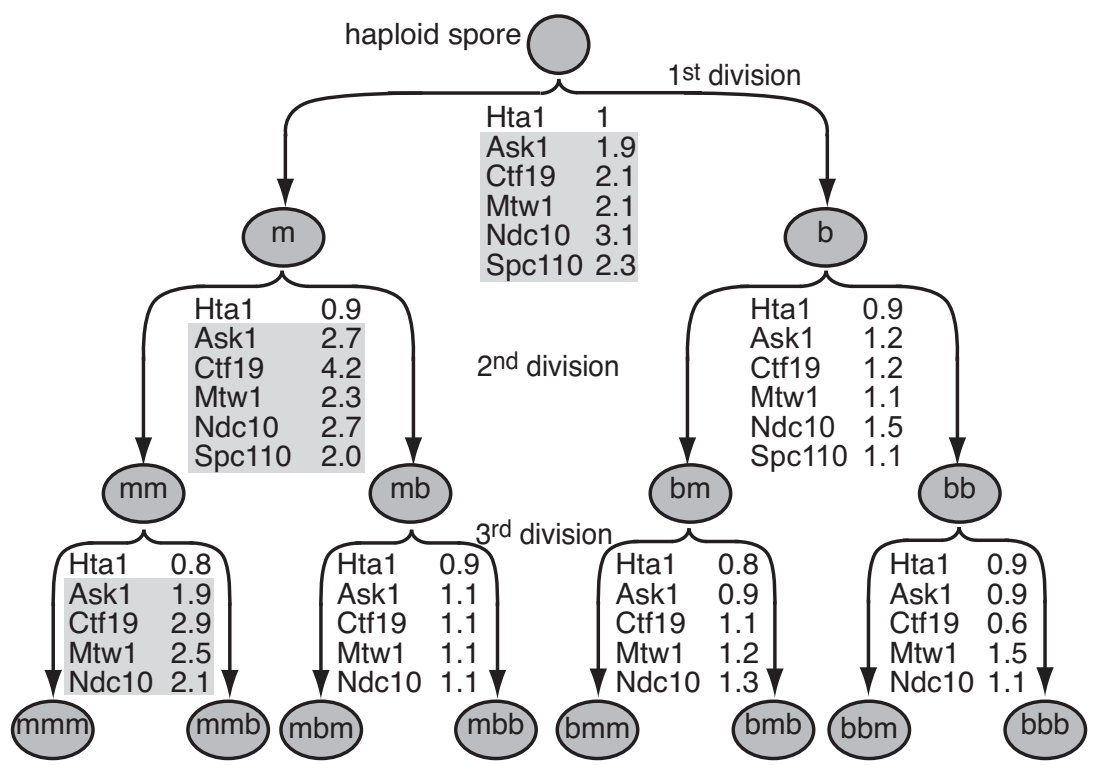

Figure 4. The mean mother-to-bud ratios $(\mathrm{m} / \mathrm{b})$ for different proteins at different postmeiotic cell divisions. The histone Hta1 divides symmetrically at each division, and the $\mathrm{m} / \mathrm{b}$ ratios are approximately 1 . In contrast, the kinetchore proteins (Ask1, Ctf19, Mtw1, and Ndc10) segregate asymmetrically only in a single mother-specific lineage derived from the spore, indicated as shaded values. Spc110, a spindle pole body protein, also shows this same pattern of asymmetry in the first two postmeiotic divisions that were measured. 
rescence microscopy to monitor protein segregation in vegetatively growing haploid and diploid cells and did not see any asymmetry within the population. Consequently, this kinetochore asymmetry defines a single postmeiotic lineage originating at the spore and maintained within the mother lineage (Fig. 4). This phenomenon bears a striking resemblance to the asymmetric division pattern of adult stem cells (see Fig. 1, top).

\section{ASYMMETRY OF SPINDLE POLE BODY SEGREGATION}

Kinetochores are attached via microtubules to the spindle pole body (SPB), the yeast microtubule-organizing center, which is a structure equivalent to that of the metazoan centrosome. Interestingly, in Drosophila, centrosome asymmetry has been characterized in specific stem cell lineages (Rebollo et al. 2007; Rusan and Peifer 2007; Yamashita et al. 2007). In the latter study, Fuller and colleagues found that by labeling old pericentrin-AKAP450 centrosomal targeting (PACT) domain protein, mother centriole pairs tend to remain in male Drosophila germ cells during cell division. Therefore, we asked whether Spc110, a PACT domain SPB protein, segregates asymmetrically in the postmeiotic lineage in a manner similar to that seen in kinetochore proteins. In yeast, components of the SPB normally segregate asymmetrically in vegetatively growing haploid cells via a Cdc28-dependent mechanism (Pereira et al. 2001; Liakopoulos et al. 2003). SPBs replicate conservatively before mitosis, with the old SPB usually segregating to the bud during cell division. However, instead of the asymmetric pattern of segregation normally found in vegetatively growing cells, we see a pattern of segregation in spores that mirrors that of the kinetochore proteins (Fig. 4). So far, we have only examined the first two postmeiotic divisions for this protein and thus we do not yet know when the standard asymmetric pattern becomes established. However, it is interesting to note that the only time in the yeast life cycle when the SPB divides without an accompanying cycle of DNA replication is during meiosis II. The SPB asymmetry seen in haploid vegetatively growing cells is associated with Cdc28-dependent cell cycle progression (Liakopoulos et al. 2003). Thus, the atypical cell cycle during meiosis II may account for a disruption in the normal pattern of SPB asymmetry. It should also be noted that the W303 yeast strain used for these studies carries a mutation in BUD4, a gene involved in bud site selection (Voth et al. 2005) that may also disrupt the normal SPB segregation phenotype.

\section{A MECHANISM TO SEGREGATE IMMORTAL DNA STRANDS}

From our studies, we have uncovered a lineage-specific pattern of asymmetry in yeast. This phenotype mimics the divisions seen in adult stem cells and thus provides a new model with which to study asymmetric stem cell divisions. Perhaps more importantly, the asymmetric segregation of kinetochore proteins offers a possible mechanism to direct the nonrandom segregation of sister centromeres (and perhaps chromatids) to a single lineage.
It is clear that the two complementary strands of a DNA molecule are not equivalent. For example, only one strand of the DNA serves as a template for transcription of a given gene. We arbitrarily refer to the two strands of the DNA molecule as Watson and Crick. After DNA replication, the old (parental) Watson and Crick strands segregate into different daughter cells (Taylor et al. 1957; Meselson and Stahl 1958). The two newly synthesized DNA strands are complementary to the old strands; hence, the two sister chromatids have largely identical sequences. The segregation of the resulting sister chromatids is therefore unimportant because no genetic differences exist between the two chromatids; i.e., unless the old strands of DNA are different from the new, or to put it another way, the old Watson is different from the new Watson.

The distinction between old and new DNA strands, based on epigenetic marks, is well established. The dramatic importance of DNA methylation first became apparent through studies done with bacteriophage. Bacterial DNA methylation protects the "old" bacterial genome from restriction endonucleases that recognize and cleave, infecting "new" unmethylated phage DNA. Phage become resistant to restriction if their DNA is methylated at the appropriate sequences. Both restricted and resistant phage share the same DNA sequence, but epigenetic methylation determines their ability to infect their host (Arber and Dussoix 1962; Arber 1965; Murray 2000).

Methylation provides a mechanism by which the cell can distinguish between old and new DNA strands after DNA replication. The newly synthesized strands are unmethylated until a methylase acts upon them. This difference in the DNA strands is exploited in bacteria to correct errors that are introduced by polymerases into the newly synthesized strands during replication. After replication, the cells are able to differentiate between the new and old strands, allowing them to correct errors using only the old strand as a template. In bacteria, the temporary hemimethylated pattern of newly synthesized DNA signals that the methylated strand is the appropriate template for mismatch repair (Glickman and Radman 1980; Modrich and Lahue 1996). In eukaryotes, the nicks created by discontinuous DNA synthesis, particularly on the lagging strand, likely serve as a guide for the mismatch repair machinery (Kunkel and Erie 2005; Modrich 2006).

Another instance of DNA strand distinction occurs in fission yeast, because the pattern of mating-type switching is determined by an epigenetic mark on one strand of the DNA (Arcangioli et al. 2007; Klar 2007). This DNA strand difference is established by the direction of progression of the replication fork (which itself is asymmetric, i.e., leading- and lagging-strand synthesis), resulting in only one of the daughter cells containing the strand imprint. Only at the next division does one of the granddaughter cells switch mating type via a recombinationdependent mechanism.

Despite mismatch repair activity, it is possible for replication errors to persist and give rise to mutations within the genome. To counter such an accumulation of mutations in a defined lineage of stem cells, John Cairns (1975) hypothesized that stem cells could retain the old 
Watson strand of DNA from each chromosome at each cell division (illustrated in Fig. 5, top). In this way, an ancestral strand of DNA from each chromosome would be retained in a single cell from one generation to the next; hence, the term for this model: the "immortal strand hypothesis." On the basis of this mechanism, replication errors would be passed on to progenitor cells, but the stem cell would always retain the original, unaltered Watson strand of DNA.

For such a model to be correct, a number of cellular requirements would have to be met. First, the cell would need a way to mark and detect the old DNA strand. Second, the cells would have to undergo an asymmetric cell division in which each chromatid that contains an ancestral DNA strand is segregated to the stem cell lineage. Finally, genetic recombination, primarily sister-chromatid exchange (SCE), would have to be suppressed to prevent the old and new DNA strands from becoming mixed.

\section{TESTING THE IMMORTAL STRAND HYPOTHESIS}

An obvious approach to test this hypothesis is to label the DNA (akin to the 1958 Meselson and Stahl experiment) and assay for retention of label in a single lineage. At the time when the immortal strand hypothesis was initially proposed, however, there was no method available to isolate pure populations of adult stem cells. Nevertheless, there was some evidence to support selective strand segregation in a number of organisms (Lark et al. 1966; Rosenberger and Kessel 1968; Priest and Shikes 1970). For example, using tritiated thymidine labeling in mouse epithelia of the tongue or intestine, it was possible to find retention of the labeled DNA in daughter cells in regions where stem cells resided (Potten et al. 1978). Experiments in budding yeast also identified nonrandom retention of labeled DNA in daughter cells (Williamson and Fennell 1981). However, a later experiment using the thymidine

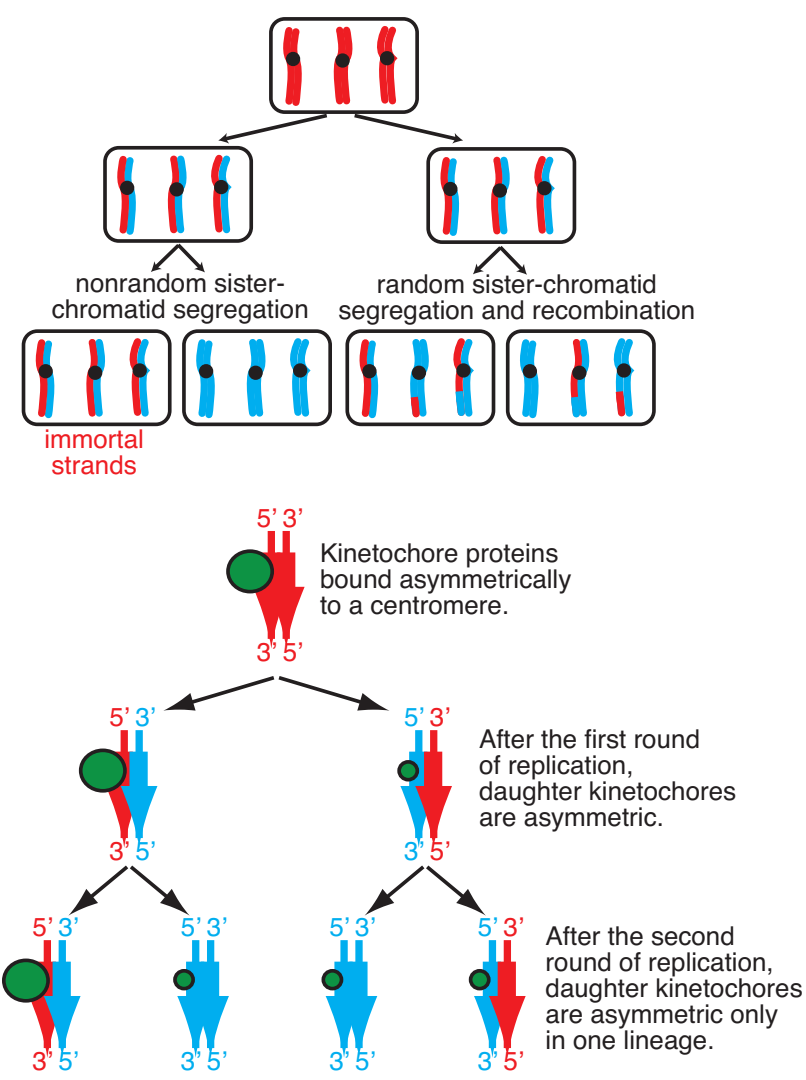

Figure 5. The immortal strand hypothesis and model for kinetochore asymmetry. (Top) The immortal strand hypothesis proposes that one original DNA strand of each chromosome segregates in a single lineage. A hypothetical haploid cell is displayed with three chromosomes each shown as double-stranded DNA. (Red) Initial DNA strands; (blue) newly synthesized DNA. If the hypothesis is correct, the original $(\mathrm{red})$ strands will be cosegregated to a single lineage of (stem) cells (lower left). If not, unbiased chromatid segregation and recombination will randomly dilute DNA strands into progeny cells (lower right). (Bottom) Kinetochore sequences have the potential to direct strand asymmetry, because yeast centromeres can be considered to be unidirectional (red arrows). If a unique kinetochore structure (large green circle) was laid down on centromeres in meiosis, all of the chromosomes containing this structure could segregate to a single lineage in subsequent postmeiotic divisions. New kinetochore structures are indicated as small green circles bound to newly synthesized centromeric DNA (blue arrows) and these segregate randomly. This model provides an explanation for the pattern of kinetochore asymmetry that we find in yeast spores. Furthermore, this model could provide a means to segregate original centromeric DNA (red) to a single lineage, whereas newly synthesized DNA (blue) segregates randomly. 
analog, bromodeoxyuridine as a DNA label, did not identify any asymmetry (Neff and Burke 1991).

Along with the ability to identify and study individual stem cell lines have come new tests of the immortal strand hypothesis, although these are not without their own shortcomings. One of the problems with any labeling strategy is that if cells do not divide, they will retain the DNA label irrespective of the segregation pattern of their DNA strands. Some recent studies have used independent means to verify that label-retaining cells are undergoing division (Conboy et al. 2007; Kiel et al. 2007). To date, the results of these studies have been mixed, with some groups reporting asymmetry, whereas others have found evidence to support a symmetric pattern of segregation; these data are reviewed elsewhere (Lansdorp 2007; Rando 2007). Alternatively, it is possible that not all of the chromosomes show selective strand segregation, in which case, a DNAlabeling strategy would be inconclusive. Indeed, there is genetic evidence in mouse stem cells for a chromosome7-specific segregation pattern (Armakolas and Klar 2006).

Our observation of an asymmetrically dividing kinetochore in a single lineage of cells provides a means to test the selective segregation of sister chromatids. An underlying assumption is that centromere sequences direct strand asymmetry by binding to kinetochore proteins unidirectionally. We suspect that once the determinant of the asymmetry is established, it persists through subsequent cell divisions as illustrated in Figure 5 (bottom). The asymmetry may be determined by specific epigenetic marks on the centromeric DNA or it could be due to strand-specific binding of the kinetochore proteins themselves. It is possible that the direction of DNA replication through the centromere establishes a strand-specific imprint (Lew et al. 2008). A precedent for this latter type of imprinting exists in fission yeast matingtype switching (Dalgaard and Klar 1999). In any event, we predict that the cell "senses" orientation of the centromeres to facilitate asymmetric segregation. We are currently testing this model by engineering a dicentric chromosome. In yeast, dicentric chromosomes can be created and maintained by using a conditional centromere that can be reversibly activated (Hill and Bloom 1989). In vegetatively growing haploid cells, the two centromeres act independently: They either cosegregate to one daughter, resulting in a normal mitosis, or, they segregate to separate daughter cells, resulting in anaphase bridges and chromosome breakage (Brock and Bloom 1994). If the orientation of the centromere is the critical determinant of asymmetry, then in the postmeiotic mother lineage, cooriented centromeres on a dicentric chromosome will always cosegregate and not result in chromosome breakage (Fig. 6, left). Whereas if the orientation of one centromere is reversed resulting in opposing orientations, then chromosome breakage should occur in each asymmetric division (Fig. 6, right).

It is worth mentioning that the asymmetric segregation of kinetochore proteins in the postmeiotic mother lineage is not found in $100 \%$ of yeast spores. The frequency of kinetochore/spindle asymmetry is $45-69 \%$ in the mother lineage compared with $7-15 \%$ in the other lineages (Fig. 7). This variation may indicate that not all yeast spores behave the same way in terms of postmeiotic asymmetry. Indeed, it is possible that only two yeast spores from each tetrad show the asymmetric phenotype. This could be related to an unusual pattern of SPB asymmetry that is found during meiosis and results in the four spores of each tetrad having nonequivalent SPBs (Taxis et al. 2005).

\section{IMMORTAL STRANDS ARE UNLIKELY TO BE FOUND IN YEAST}

At this time, we have no evidence to suggest that the postmeiotic mother lineage selectively segregates DNA strands. Indeed, even if it did, the mother lineage in yeast is mortal; it ages and senesces after approximately 30-40 divisions (Mortimer and Johnston 1959). Furthermore, SCE would need to be suppressed in the mother lineage because SCE would result in crossing-over of the two sis-

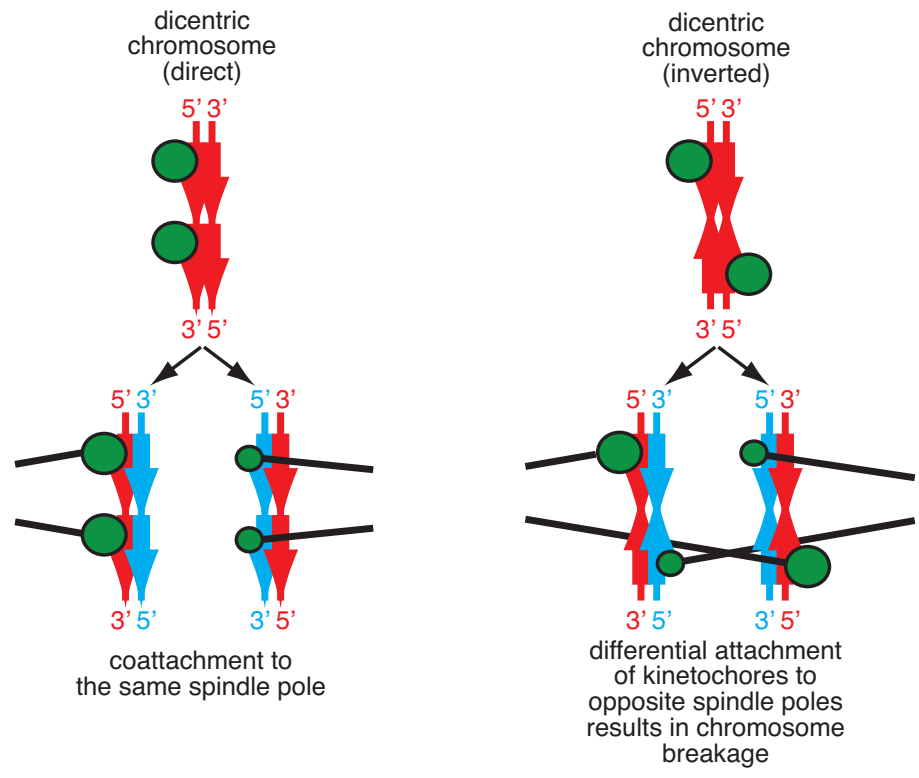

Figure 6. A dicentric chromosome test of nonrandom segregation. As in Figure 5 (bottom), a unique kinetochore complex is established on the centromeres unidirectionally in meiosis (large green circle). (Left) DNA replication of a direct dicentric chromosome (top) results in two chromatids, each of which containing both old (red) and new (blue) DNA strands (below). The meiotically induced kinetochore complexes are on the same sister chromatid after replication. Cosegregation of these complexes to a single postmeiotic mother lineage results in cosegregation of coaligned centromeres (indicated by the orientation of spindle microtubules; black lines). (Right) However, if the centromere sequences are in inverted orientations, the meiosis-induced kinetochore structure will be bound to separate sister chromatids after DNA replication. In this case, if these kinetochore complexes cosegregate, both of the dicentric chromatids will likely break. 


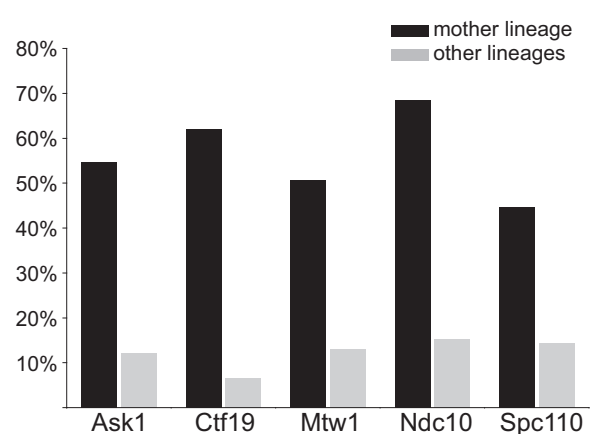

Figure 7. Proportion of asymmetric divisions for each of the asymmetrically dividing proteins that we studied. The proportion (\%) of asymmetric divisions (defined as an $\mathrm{m} / \mathrm{b}$ ratio greater than 1.6) is shown for the mother lineage (black) and the other lineages (gray).

ter-chromatid arms, thereby scrambling the old and new DNA strands in each chromatid (Fig. 5, top). Although SCE is difficult to measure, it is thought to occur frequently during $G_{2}$ and $M$ phases in yeast (Kadyk and Hartwell 1992; Zou and Rothstein 1997; Gonzalez-Barrera et al. 2003). A reasonable surrogate for measuring SCE is the appearance of a DNA-repair focus that arises spontaneously during S phase (Lisby et al. 2001, 2003). We reasoned that if SCE were repressed, we might see suppression of DNA recombination foci in dividing spores and the mother cells derived from them. However, Rad52 foci do appear in these cells, indicating that recombination is not completely inactive (P. Thorpe et al., unpubl.). Together, these data argue against an immortal strand in postmeiotic yeast. However, kineotchore-driven asymmetry could selectively segregate centromeres and their closely linked sequences in the single postmeiotic lineage.

\section{SELECTIVE CENTROMERE SEGREGATION}

The selective segregation of centromere sequences could have an important role in speciation. For example, in metazoan diploids where the two parental species are diverging, each set of centromeres may have different efficiencies of kinetochore binding (Henikoff and Malik 2002). This difference is due to the coevolution of the centromere sequence together with its kinetochore-binding protein, thereby necessitating cosegregation of these components. During female meiosis, only one of the four products forms a gamete, and the other three polar bodies are lost. Hence, there is an opportunity to select for the cosegregation of one parental set of centromeres and its kinetochore-binding protein, a process akin to meiotic drive (Sandler and Novitski 1957; Henikoff et al. 2001; Pardo-Manuel de Villena and Sapienza 2001; Copenhaver 2004). Surprisingly, it appears that yeast centromeres are also evolving rapidly despite the absence of meiotic drive, because all four meiotic products are viable in yeast (Bensasson et al. 2008). Thus, the kinetochore asymmetry described here may enable a single lineage derived from a spore to select for "preferred" centromere sequences with a high affinity for their kinetochore-binding proteins. This in turn may explain the divergence of yeast centromere sequences. Therefore, it is possible that the kinetochore asymmetry described here is involved in promoting evolutionary divergence. Yeast provides one of the few organisms in which such a model could be tested practically.

\section{CONCLUSIONS AND PERSPECTIVES}

Yeast is a powerful model system due in part to a large array of experimental techniques and has consequently been used to characterize many basic cell biological pathways, such as those involved in cell cycle control and genetic recombination. We have characterized a lineage-specific pattern of asymmetry that mimics that seen in adult stem cells. This type of asymmetric division is now amenable to study in yeast. A first step toward further study of this phenomenon will be a highthroughput microscopy screen to identify the genes that are required for this postmeiotic asymmetric pattern. This set of genes is likely to include those that control the lineage-specific asymmetric segregation pattern of the yeast kinetochore. We predict that these will be conserved and will be key regulators of the pattern of asymmetric division that occurs during development and in adult stem cells.

\section{ACKNOWLEDGMENTS}

We thank Argiris Efstratiadis and Rudy Leibel for motivating our interest in cellular asymmetry. Phil Heiter, Trisha Davis, Kerry Bloom, Tim Bestor, Kara Bernstein, Michael Lisby, Rebecca Burgess, Bob Reid, Qi Feng, and Jackie Barlow provided strains and/or helpful advice and suggestions on this manuscript. This work was supported by the Russ Berrie Diabetes Foundation and National Institutes of Health grants GM50237 and GM67055.

\section{REFERENCES}

Arber, W. 1965. Host specificity of DNA produced by Escherichia coli. V. The role of methionine in the production of host specificity. J. Mol. Biol. 11: 247-256.

Arber, W. and Dussoix, D. 1962. Host specificity of DNA produced by Escherichia coli. I. Host controlled modification of bacteriophage ג. J. Mol. Biol. 5: 18-36.

Arcangioli, B., Roseaulin, L., and Holmes, A. 2007. Mating-type switching in S. pombe. In Molecular genetics of recombination (ed. A. Aguilera and R. Rothstein), pp. 251-275. SpingerVerlag, Berlin.

Armakolas, A. and Klar, A.J. 2006. Cell type regulates selective segregation of mouse chromosome 7 DNA strands in mitosis. Science 311: 1146-1149.

Bensasson, D., Zarowiecki, M., Burt, A., and Koufopanou, V. 2008. Rapid evolution of yeast centromeres in the absence of drive. Genetics 178: 2161-2167.

Bouck, D. and Bloom, K. 2005. The role of centromere-binding factor $3(\mathrm{CBF} 3)$ in spindle stability, cytokinesis, and kinetochore attachment. Biochem. Cell Biol. 83: 696-702.

Brock, J.A. and Bloom, K. 1994. A chromosome breakage assay to monitor mitotic forces in budding yeast. J. Cell Sci. 107: 891-902.

Cairns, J. 1975. Mutation selection and the natural history of cancer. Nature 255: 197-200.

Cheeseman, I.M., Brew, C., Wolyniak, M., Desai, A., Anderson, S., Muster, N., Yates, J.R., Huffaker, T.C., Drubin, D.G., and Barnes, 
G. 2001. Implication of a novel multiprotein Dam1p complex in outer kinetochore function. J. Cell Biol. 155: 1137-1145.

Conboy, M.J., Karasov, A.O., and Rando, T.A. 2007. High incidence of nonrandom template strand segregation and asymmetric fate determination in dividing stem cells and their progeny. PLoS Biol. 5: e102.

Copenhaver, G.P. 2004. Who's driving the centromere? J. Biol. 3: 17.

Dalgaard, J.Z. and Klar, A.J. 1999. Orientation of DNA replication establishes mating-type switching pattern in $S$. pombe. Nature 400: 181-184.

De Wulf, P., McAinsh, A.D., and Sorger, P.K. 2003. Hierarchical assembly of the budding yeast kinetochore from multiple subcomplexes. Genes Dev. 17: 2902-2921.

Glickman, B.W. and Radman, M. 1980. Escherichia coli mutator mutants deficient in methylation-instructed DNA mismatch correction. Proc. Natl. Acad. Sci. 77: 1063-1067.

Gonzalez-Barrera, S., Cortes-Ledesma, F., Wellinger, R.E., and Aguilera, A. 2003. Equal sister chromatid exchange is a major mechanism of double-strand break repair in yeast. Mol. Cell 11: $1661-1671$.

Henikoff, S. and Malik, H.S. 2002. Centromeres: Selfish drivers. Nature 417: 227.

Henikoff, S., Ahmad, K., and Malik, H.S. 2001. The centromere paradox: Stable inheritance with rapidly evolving DNA. Science 293: 1098-1102.

Hill, A. and Bloom, K. 1989. Acquisition and processing of a conditional dicentric chromosome in Saccharomyces cerevisiae. Mol. Cell. Biol. 9: 1368-1370.

Joglekar, A.P., Bouck, D.C., Molk, J.N., Bloom, K.S., and Salmon, E.D. 2006. Molecular architecture of a kinetochoremicrotubule attachment site. Nat. Cell Biol. 8: 581-585.

Kadyk, L.C. and Hartwell, L.H. 1992. Sister chromatids are preferred over homologs as substrates for recombinational repair in Saccharomyces cerevisiae. Genetics 132: 387-402.

Kiel, M.J., He, S., Ashkenazi, R., Gentry, S.N., Teta, M., Kushner, J.A., Jackson, T.L., and Morrison, S.J. 2007. Haematopoietic stem cells do not asymmetrically segregate chromosomes or retain BrdU. Nature 449: 238-242.

Klar, A.J. 2007. Lessons learned from studies of fission yeast mating-type switching and silencing. Annu. Rev. Genet. 41: 213-236.

Kunkel, T.A. and Erie, D.A. 2005. DNA mismatch repair. Аnnu. Rev. Biochem. 74: 681-710.

Lansdorp, P.M. 2007. Immortal strands? Give me a break. Cell 129: $1244-1247$.

Lark, K.G., Consigli, R.A., and Minocha, H.C. 1966. Segregation of sister chromatids in mammalian cells. Science 154: 12021205.

Lew, D.J., Burke, D.J., and Dutta, A. 2008. The immortal strand hypothesis: How could it work? Cell 133: 21-23.

Liakopoulos, D., Kusch, J., Grava, S., Vogel, J., and Barral, Y. 2003. Asymmetric loading of Kar9 onto spindle poles and microtubules ensures proper spindle alignment. Cell 112: $561-574$.

Lisby, M., Rothstein, R., and Mortensen, U.H. 2001. Rad52 forms DNA repair and recombination centers during $\mathrm{S}$ phase. Proc. Natl. Acad. Sci. 98: 8276-8282.

Lisby, M., Mortensen, U.H., and Rothstein, R. 2003. Colocalization of multiple DNA double-strand breaks at a single Rad52 repair centre. Nat. Cell Biol. 5: 572-577.

Long, R.M., Singer, R.H., Meng, X., Gonzalez, I., Nasmyth, K., and Jansen, R.P. 1997. Mating type switching in yeast controlled by asymmetric localization of $A S H 1$ mRNA. Science 277: 383-387.

Meselson, M. and Stahl, F.W. 1958. The replication of DNA in
Escherichia coli. Proc. Natl. Acad. Sci. 44: 671-682.

Modrich, P. 2006. Mechanisms in eukaryotic mismatch repair. $J$. Biol. Chem. 281: 30305-30309.

Modrich, P. and Lahue, R. 1996. Mismatch repair in replication fidelity, genetic recombination, and cancer biology. Annu. Rev. Biochem. 65: 101-133.

Mortimer, R.K. and Johnston, J.R. 1959. Life span of individual yeast cells. Nature 183: 1751-1752.

Murray, N.E. 2000. Type I restriction systems: Sophisticated molecular machines (a legacy of Bertani and Weigle). Microbiol. Mol. Biol. Rev. 64: 412-434.

Neff, M.W. and Burke, D.J. 1991. Random segregation of chromatids at mitosis in Saccharomyces cerevisiae. Genetics 127: 463-473.

Nekrasov, V.S., Smith, M.A., Peak-Chew, S., and Kilmartin, J.V. 2003. Interactions between centromere complexes in Saccharomyces cerevisiae. Mol. Biol. Cell 14: 4931-4946.

Pardo-Manuel de Villena, F. and Sapienza, C. 2001. Female meiosis drives karyotypic evolution in mammals. Genetics 159: $1179-1189$

Pereira, G., Tanaka, T.U., Nasmyth, K., and Schiebel, E. 2001. Modes of spindle pole body inheritance and segregation of the Bfa1p-Bub2p checkpoint protein complex. EMBO J. 20: 6359-6370.

Pinsky, B.A., Tatsutani, S.Y., Collins, K.A., and Biggins, S. 2003. An Mtw1 complex promotes kinetochore biorientation that is monitored by the Ipl1/Aurora protein kinase. Dev. Cell 5: 735-745.

Potten, C.S., Hume, W.J., Reid, P., and Cairns, J. 1978. The segregation of DNA in epithelial stem cells. Cell 15: 899-906.

Priest, J.H. and Shikes, R.H. 1970. Distribution of labeled chromatin. I. M 1 and M 2 anaphases of diploid and tetraploid cultured mammalian cells. J. Cell Biol. 47: 99-106.

Rando, T.A. 2007. The immortal strand hypothesis: Segregation and reconstruction. Cell 129: 1239-1243.

Rebollo, E., Sampaio, P., Januschke, J., Llamazares, S., Varmark, H., and Gonzalez, C. 2007. Functionally unequal centrosomes drive spindle orientation in asymmetrically dividing Drosophila neural stem cells. Dev. Cell 12: 467-474.

Rosenberger, R.F. and Kessel, M. 1968. Nonrandom sister chromatid segregation and nuclear migration in hyphae of Aspergillus nidulans. J. Bacteriol. 96: 1208-1213.

Rusan, N.M. and Peifer, M. 2007. A role for a novel centrosome cycle in asymmetric cell division. J. Cell Biol. 177: 13-20.

Sandler, I. and Novitski, E. 1957. Meiotic drive as an evolutionary force. Am. Nat. 91: 105-110.

Taxis, C., Keller, P., Kavagiou, Z., Jensen, L.J., Colombelli, J., Bork, P., Stelzer, E.H., and Knop, M. 2005. Spore number control and breeding in Saccharomyces cerevisiae: A key role for a self-organizing system. J. Cell Biol. 171: 627-640.

Taylor, J.H., Woods, P.S., and Hughes, W.L. 1957. The organization and duplication of chromosomes as revealed by autoradiographic studies using tritium-labeled thymidine. Proc. Natl. Acad. Sci. 43: 122-128.

Voth, W.P., Olsen, A.E., Sbia, M., Freedman, K.H., and Stillman, D.J. 2005. $A C E 2, C B K 1$, and BUD4 in budding and cell separation. Eukaryot. Cell 4: 1018-1028.

Williamson, D.H. and Fennell, D.J. 1981. Nonrandom assortment of sister chromatids in yeast mitosis. Alfred Benzon Symp. 16: 89-107.

Yamashita, Y.M., Mahowald, A.P., Perlin, J.R., and Fuller, M.T. 2007. Asymmetric inheritance of mother versus daughter centrosome in stem cell division. Science 315: 518-521.

Zou, H. and Rothstein, R. 1997. Holliday junctions accumulate in replication mutants via a RecA homolog-independent mechanism. Cell 90: 87-96. 


\title{
$8_{\mathrm{CSH}}^{\infty} \mathrm{C}$ Cold Spring Harbor Symposia SYMPOSIA on Quantitative Biology
}

\section{Modeling Stem Cell Asymmetry in Yeast}

\author{
P.H. Thorpe, J. Bruno and R. Rothstein
}

Cold Spring Harb Symp Quant Biol 2008 73: 81-88 originally published online November 6, 2008 Access the most recent version at doi:10.1101/sqb.2008.73.010 $\begin{array}{ll}\text { References } & \begin{array}{l}\text { This article cites } 52 \text { articles, } 26 \text { of which can be accessed free at: } \\ \text { http://symposium.cshlp.org/content/73/81.full.html\#ref-list-1 }\end{array}\end{array}$

\section{License}

Email Alerting Receive free email alerts when new articles cite this article - sign up in the box at the Service top right corner of the article or click here. 